\title{
El proyecto de la fortaleza de San Fernando de Figueras. Una aportación al conocimiento de la dirección interina de Juan Martín Cermeño al frente de los ingenieros reales
}

\author{
Pablo De la Fuente de Pablo
}

El período que dista entre 1749 y 1756, época en la cual Juan Martín Cermeño asume la comandancia general de los ingenieros reales y que recoge a "grosso modo» la mayor parte del reinado de Fernando $\mathrm{VI}$, creemos que es uno de los períodos más interesantes de la ingeniería militar española de todo el siglo XVIII, siendo en particular el proyecto de la fortaleza de San Fernando de Figueras uno de los hitos significativos de la historia de dicho cuerpo.

Después del corto mandato del marqués de Pozoblanco, en 1749 se inicia - y no sin polémica, tal como veremos- un primer período de aproximadamente 7 años durante el cual Juan Martín Cermeño asumirá la dirección del cuerpo de ingenieros. Una primera consideración a este respecto es resaltar la magnífica aproximación al tema que realiza Marzal en su excelente trabajo '. Siendo éste nuestro punto de partida, cabe considerar que durante la dirección de este personaje se abordarán importantes reformas organizativas y se ejecutarán proyectos de gran importancia.

Juan Martín Cermeño nace en Ciudad Rodrigo en la última década del siglo XVII. En 1719 ingresa en los ingenieros reales, participando en 1727 en el asedio de Gibraltar. Durante los años treinta son destacables

1 Sobre este punto consúltese A. Marzal Martinez: La ingeniería militar en la España del XVII. Nuevas aportaciones a la historia de su legado científico y monumental; tes. doc. dir. J. Hernández Perera. Universidad Complutense de Madrid : Madrid, 1991, t. I, págs. 101-115. 
sus trabajos de fortificación en la costa de Málaga. En 1746 es nombrado ingeniero jefe del ejército de Italia, decisión que abre una gran polémica al ser adelantado en el escalafón a costa del ingeniero Marqueli. Nuevamente en 1749, cuando es nombrado comandante general de ingenieros, dicho nombramiento se hace a costa del ingeniero Ignacio Sala, más antiguo que Cermeño. Por esta razón, Cermeño será un hombre algo impopular entre sus compañeros. Ante esta situación, cabe destacar el carácter interino del cargo, pese a las repetidas ocasiones en que Cermeño solicitará su nombramiento en firme, interinidad que ejercerá hasta que en 1756 sea nombrado ingeniero general el conde de Aranda ${ }^{2}$. No será hasta unos años más tarde, concretamente en 1769, cuando sea nombrado ingeniero general en firme, cargo que ostentará hasta su muerte, ocurrida en Barcelona en $1773^{3}$.

En lo referente a los aspectos organizativos de la dirección interina de Cermeño, cabe significar que en lo tocante a política de personal existen una serie de importantes logros tales como la constitución del montepío o la importante reforma de la enseñanza del cuerpo con la famosa Real Ordenanza de 1751, la cual venía a consolidar la gran obra realizada hasta ese momento por Pedro de Lucuze, director de la Academia de Barcelona y pilar básico de la tratadística militar española del siglo XVIII ${ }^{4}$.

Siguiendo con la política de personal, un problema constante con el que se enfrentará Cermeño será la insuficiencia de ingenieros, que tal como Marzal explica está sobre todo en relación con la absorción de ingenieros para proyectos ajenos a los de la secretaría de guerra, básicamente obras para la armada o de infraestructura civil ${ }^{5}$.

\footnotetext{
2 El punto màs significativo del mandato del conde de Aranda será la reagrupación de los cuerpos de artillería e ingenieros. Véase A. MARZAL MARTínez, op. cit., t. I págs. 116-128.

3 Sobre la figura de Cermeño una referencia básica es J. DE LA LLAVE y GARcía: «D. Juan Martín Zermeño, Teniente General e Ingeniero general», en Revista del Memorial de Ingenieros, XXVIII 5 a . Madrid, 1911, págs. 101-164. Véase también A. MarzaL Martínez, op. cit., t. II págs. 1.189-1.191 y también H. CAPEl ET AlLli: Los ingenieros militares en España. Sigio Xvil. Repertorio biográfico e inventario de su labor cieniffica y espacial. Universidad de Barcelona: Barcelona, 1983, págs. 309-313. Con respecto a la muerte de Cermeño véase (A)RCHIVO (G)ENERAL DE (S)IMANCAS: Guerra Moderna, leg. 3.803.

" La figura de Pedro de Lucuze está intrinsecamente relacionada con la formación de los ingenieros militares de buena parte del siglo xvIII. Sobre esta interesantísima cuestión consúltese $H$. CAPEL ET Allil: De Palas a Minerva. La formación cientifica y la estructura institucional de los ingenieros militares en el siglo xvIII. Serbal, C.S.I.C., Barcelona, 1988, 390 pág. Sobre aspectos relacionados con la actuación de Lucuze al frente de la Academia de Barcelona véase A. MARZAL Martínez, op. cit., t. I págs. 304 y ss.

5 Ello es debido al hecho que no se formó el cuerpo de ingenieros de marina hasta 1771. Es evidente, tal como afirman MARZAL O CAPEL, que el hecho que los ingenieros militares tomaran un
} 
Entre las intervenciones llevadas a cabo bajo la dirección interina de este ingeniero, cabe considerar como asunto capital de este período el tema de la defensa de fronteras, siendo el problema fundamental los nuevos planteamientos relativos a la frontera con Francia. Si bien en el tema de la frontera con Portugal, ya había sido atendido parcialmente con el comienzo de los trabajos del Fuerte de La Concepción en los años treinta ${ }^{6}$, sin embargo, la frontera con Francia -en especial la del Principado de Cataluña en donde sus intervenciones son numerosísimas- requerirá de la actuación de los ingenieros reales, siendo lamentable el estado del sistema defensivo catalán, el cual se hallaba prácticamente desarticulado desde el Tratado de los Pirineos de 1659, pasando Perpiñán y la mayoría de las plazas fronterizas de la región a la soberanía del rey de Francia. Si bien es cierto que son numerosos los proyectos de fortificación del nuevo espacio limítrofe a lo largo de toda la segunda mitad del siglo XVII, lo cierto es que la plaza de Rosas - la más arrimada a la frontera- no cubría de forma eficaz el territorio.

Prueba de que la mayor preocupación de Cermeño será este punto es el hecho que éste comisiona al ingeniero Pedro de Superviela ${ }^{7}$. Sin embargo, el informe de dicho ingeniero no reviste un gran interés, sobre todo debido al hecho de no aportar nuevos elementos de juicio sobre este punto, ya que éste viene a recoger básicamente las opiniones de Verboom. Ante ello, en 1751 Cermeño comisiona a Pedro de Lucuze, del cual ya hemos hablado anteriormente, para este menester. El informe de Pedro de Lucuze a este respecto es interesantísimo, ya que la reflexión queda encerrada en un acertadísimo estudio geoestratégico del Ampurdán. El resultado del dictamen de Lucuze es que el sitio más adecuado es el lugar llamado Paso de las Molas, al norte de la villa de Figueras y sobre la margen meridional del río Muga ${ }^{8}$.

Sin embargo, Cermeño desoirá las recomendaciones de Lucuze y elaborará su propio proyecto, lo cual será acogido con cierta polémica. Sobre este punto debemos afirmar que dicha decisión será un nuevo episodio de

papel protagonista en numerosos proyectos civiles ha hecho que el estudio y conocimiento de sus actuaciones sobrepase el campo estrictamente militar.

6 Sobre esta fortificación consideramos de gran utilidad la obra de F. R. DE LA FLOR: El Fuerte de La Concepción y la arquitectura militar de los siglos XVII y XVIII. Diputación de Salamanca: Salamanca, 1987; 198 pág.

A.G.S.: Guerra Moderna, leg. 2.295.

\& A.G.S.: Guerra Moderna, leg. 2.295. Sobre el proyecto en sí, véase P. DE LA FuENTE: «Una alternativa al castell de Sant Ferran: El projecte de Pedro de Lucuze», en Revista de Girona. Girona (en prensa). 
descontento hacia Cermeño. Dejando de lado la comparación directa entre ambos proyectos, pensamos que existieron una serie de claves que explicarán dicha situación. El hecho de que el proyecto de Pedro de Lucuze fuera defendido por numerosos oficiales del cuerpo, pensamos que tiene tres posibles explicaciones. En primer lugar, creemos que una de las razones fue que la idea de Lucuze fuera considerada como más acertada intrínsecamente. También sostenemos el criterio de que el hecho que Lucuze fuera director de la Academia de Barcelona es un factor a tener en consideración. Pensamos que su acertada labor y su prestigio como docente motivó que un buen número de ingenieros - muchos de ellos antiguos alumnos de la academia - se pusieran de su lado. Por último, creemos que otra razón que explicaría la situación es que dicha polémica canalizaría el descontento hacia la figura de Cermeño, cuestión que anteriormente hemos abordado, siendo considerada la desautorización de una figura de la categoría de Lucuze como una nueva transgresión del comandante general.

En 1752, Cermeño elabora su dictamen «Relación del territorio y frontera del Ampurdán en el Principado de Cataluña y de sus entradas» 9 . Uno de los puntos más sobresalientes de dicho proyecto es el detenido estudio que este personaje realiza -remontándose a incluso en casi cien años, lo cual es prueba evidente de una importante investigación archivística- de los más significativos proyectos que hasta ese momento han sido propuestos. Sin embargo, la principal lectura de dicho informe - dejando de lado su propio proyecto- es la ausencia de espíritu crítico hacia el proyecto de Lucuze, lo cual deja entrever el poco ánimo de Cermeño a entrar en polémicas. Pese a ello, si comparamos ambos informes, vemos cómo Cermeño emite una opinión muy próxima a la de Lucuze en relación a la plaza de Rosas y su rehabilitación, lo cual contradice las opiniones de Verboom y Superviela, los cuales se manifiestan claramente por el abandono de la plaza.

Realmente la ubicación de la futura fortaleza llegó a ser un tema revestido de una fuerte polémica, que finalmente es solucionada por el capitán general de Principado en favor de la idea de Cermeño, dándose comienzo a las obras en 1753.

El lugar elegido por Cermeño era la llamada montaña de Capuchinos, un promontorio situado aproximadamente a un kilómetro al noroeste de Figueras, así denominado por la existencia de un convento de dicha orden en el lugar. Una primera observación que podemos realizar sobre la elección del lugar es que no es una idea original de Cermeño. Este hecho resalta aún

9 A.G.S.: Guerra Moderna, leg. 3.455. 
más la investigación archivística a la que anteriormente hemos aludido, ya que dicho emplazamiento ya es propuesto por Baltasar de Rojas y Pantoja en $1660^{10}$. Con respecto a la denominación de la fortaleza, es evidente que el nombre de la misma, San Fernando, refleja claramente dos aspectos. Por un lado una clara asociación al nombre y la figura del soberano reinante, y por otra, la larga tradición asentada ya claramente en el reinado de Felipe II en lo referente a las advocaciones al santoral a la hora de designar los nombres tanto de las fortalezas como sus baluartes y otros elementos defensivos ${ }^{11}$, si bien sobre este punto cabe decir que una vez iniciadas las obras, todavía no se había decidido el nombre definitivo (fig. 1).

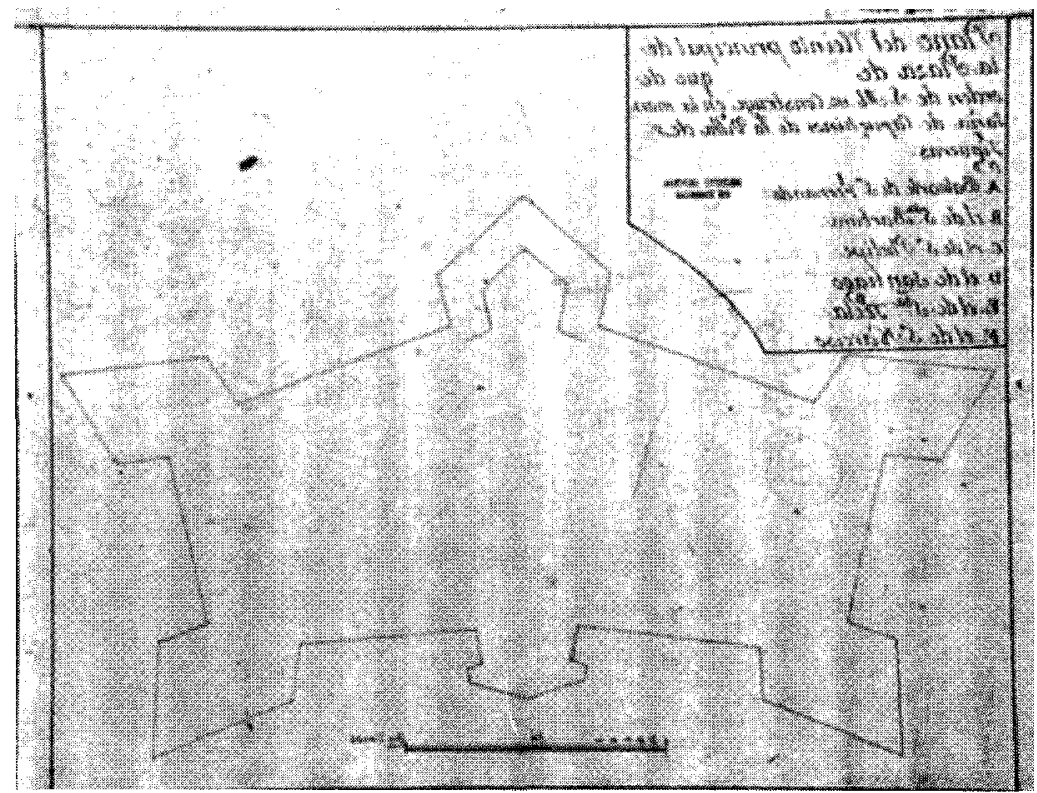

Fig. 1. Croquis del cuerpo de la plaza datado en 1753. Obsérvese como hecho más sobresaliente la ausencia de nombre de la fortaleza y cómo uno de los baluartes es llamado de San Fernando, lo cual hará que cuando la plaza sea posteriormente bautizada con este nombre dicho baluarte pase a llamarse de San Dalmacio (A.G.S.: Mapas, Planos y Dibujos, XVIII-72).

10 A.G.S.: Guerra Antigua, leg. 1.950. Véase también P. DE LA FuENTE, op. cit.

11 A. CÁmARA Muñoz: «La fortificación de la monarquía de Felipe ll», en Espacio, Tiempo y Forma, $\mathrm{H}^{\mathrm{a}}$ del Arte 2, Serie VII. Universidad Nacional de Educación a Distancia. Madrid, 1989, págs. 77-78. 
El proyecto en cuestión cabe calificarlo como una empresa de gran magnitud ${ }^{12}$. La previsión del mismo era la ejecución de una obra de fortificación capaz para 10.000 hombres y 500 caballos, lo cual sobrepasaba considerablemente las previsiones de cualquier proyecto anterior. Si a ello sumamos el alto coste que suponía el desmonte y terraplenamiento de la montaña a fin de asentar las obras, podemos comprender fácilmente que el montante financiero del proyecto fuera de gran envergadura, ascendiendo solamente las obras de fortificación a más de 20 millones de reales de vellón ${ }^{13}$. Los edificios internos de la plaza ni entraban en la relación ni se habían proyectado aún.

La plaza de San Fernando (fig. 2) -de un perímetro total de 3.120 metros a la altura del camino cubierto- se compone básicamente del cuerpo de la misma (figs. 1 y 2), de estructura pentagonal y formado por seis baluartes, uno de ellos plano. Por lo que respecta a las obras exteriores, éstas se componen de tres hornabeques o medias-coronas, siete mediaslunas o revellines - tres de ellos en vanguardia de los hornabeques- y dos contraguardias.

Los detalles más sobresalientes del cuerpo de la plaza son el caballero del baluarte de Santa Bárbara y el baluarte plano de Santa Tecla, elementos defensivos situados a poniente y levante respectivamente.

Piespecto a las obras exteriores, debemos significar que todos los sectores -excepto el de levante guarnecido únicamente por dos revellinesestaban cubiertos por hornabeques coronados con sendos revellines. Además, el perímetro entre los tres hornabeques se cerraba mediante una sucesión de revellines y contraguardias.

Respecto de los accesos a la plaza, el acceso principal se realizaba a partir del ala meridional del hornabeque de San Roque, en el cual se

\footnotetext{
12 Hasta este momento, la aportación más interesante realizada es la de C. DíAz CAPMANY: El castillo de San Fernando de Figueres. Su historia. Generalitat de Catalunya, Barcelona, 1982, 200 pág. El contenido de la obra trata del devenir histórico de dicha fortificación desde su construcción hasta nuestros dias. Vista la amplia horquilla cronológica, los aspectos relacionados con la discusión sobre el emplazamiento de la fortaleza y su construcción se resumen básicamente en 3 capítulos - unas 45 páginas-cuyo contenido global es aproximadamente el de un artículo largo. Además cabe considerar seriamente el hecho que si bien es cierto que hoy por hoy es lo mejor escrito sobre el tema, el trabajo presenta graves taras tanto desde el punto de vista del rigor metodológico como de la linealidad de la exposición. Es por ello que concluimos que tanto el presente artículo - el cual pretende replantear ciertas cuestiones así como aportar nuevos datos - como la obra de Díaz son aportaciones no concluyentes sobre la cuestión. Es evidente que sólo la construcción - con todas sus implicaciones- de tan importante fortaleza requiere un profundo y exhaustivo estudio que no se ha hecho hasta el momento.

13 C. Díz Capmany: op. cit., págs. 31 y 34.
} 


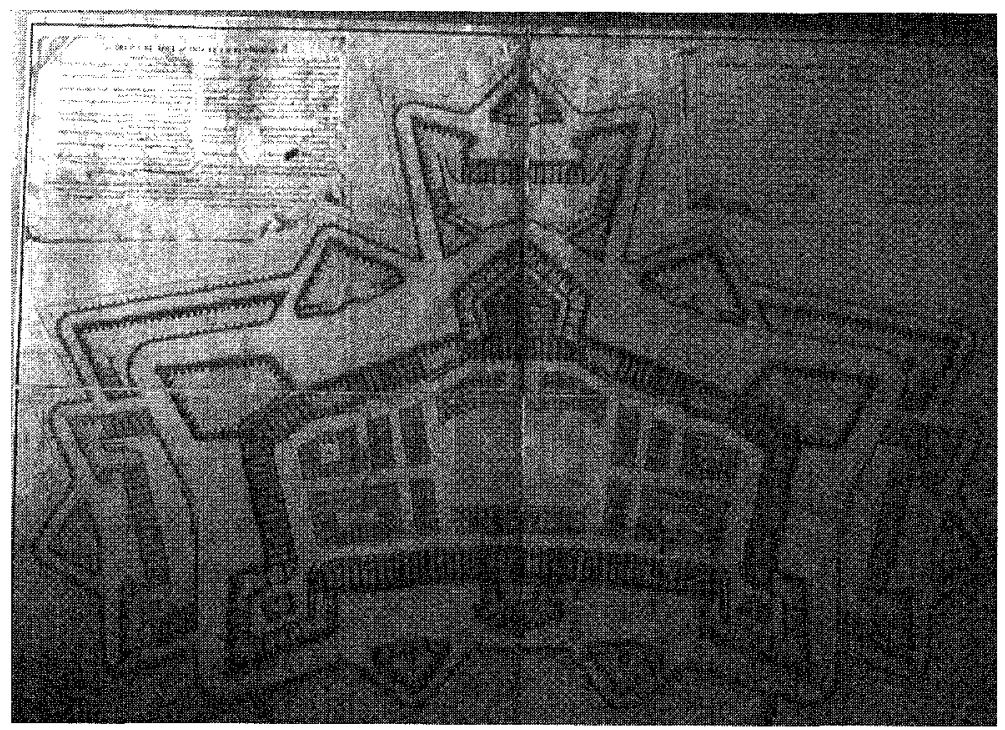

Fig. 2. Plano realizado por Pedro Martín-Paredes Cermeño, hijo de Juan Martín Cermeño e ingeniero participante activo en las obras de la fortaleza, datado en 1760 ((S)ervicio (G)eográfico del (E)jército: Cartoteca Histórica, $n^{\circ} 611$ ).

dispone un puente levadizo y una puerta en la cual no faltan motivos ornamentales. Una vez atravesada esta obra exterior, el acceso al cuerpo de la plaza se realizaba mediante un viaducto elevado respecto del foso perpendicular a la cortina entre los baluartes de San Narciso y San Dalmacio. En el centro de dicha cortina se hallaba la puerta principal de la plaza — de inspiración neoclásica- protegida por un puente levadizo. Tanto la puerta inscrita en el recinto exterior como la interior, dan buena prueba de la sólida formación arquitectónica de los ingenieros militares, siendo este tipo de elementos los únicos en los cuales se puede apreciar alguna creatividad, visto el carácter estrictamente funcional de la obra. Es evidente que la comunicación principal de la fortaleza estaba orientada hacia la villa de Figueras, principal núcleo urbano de los alrededores y cabeza de la comarca ampurdanesa (fig. 4).

Un aspecto importante a destacar es el aprovechamiento que se realiza de los elementos defensivos para la ejecución de diversas obras tales como cuarteles, almacenes, depósitos, polvorines y caballerizas en los espacios comprendidos entre la escarpa y su contramuro, siendo los ejemplos en este punto numerosísimos, destacando las técnicas de blindaje empleadas para estos elementos (fig. 3). De entre todos ellos podemos 


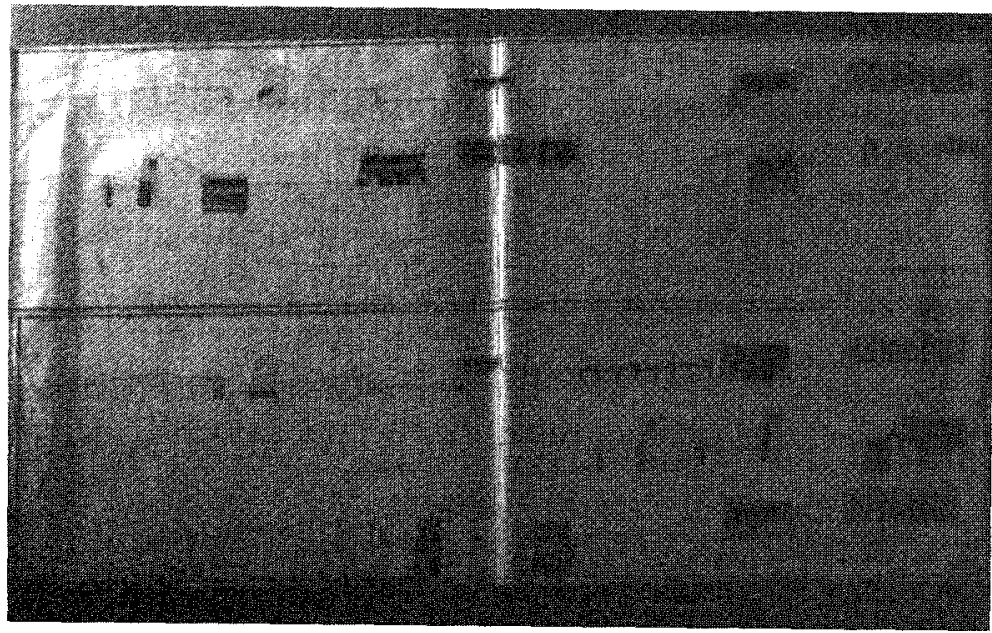

Fig. 3. Perfiles relativos al plano de la figura 2. Obsérvese como detalle más significativo el reaprovechamiento del espacio interno de los elementos defensivos, en donde destacan las cisternas y los abovedados a prueba de bomba (S.G.E.: Cartoteca Histórica, $n^{\circ} 612$ ).

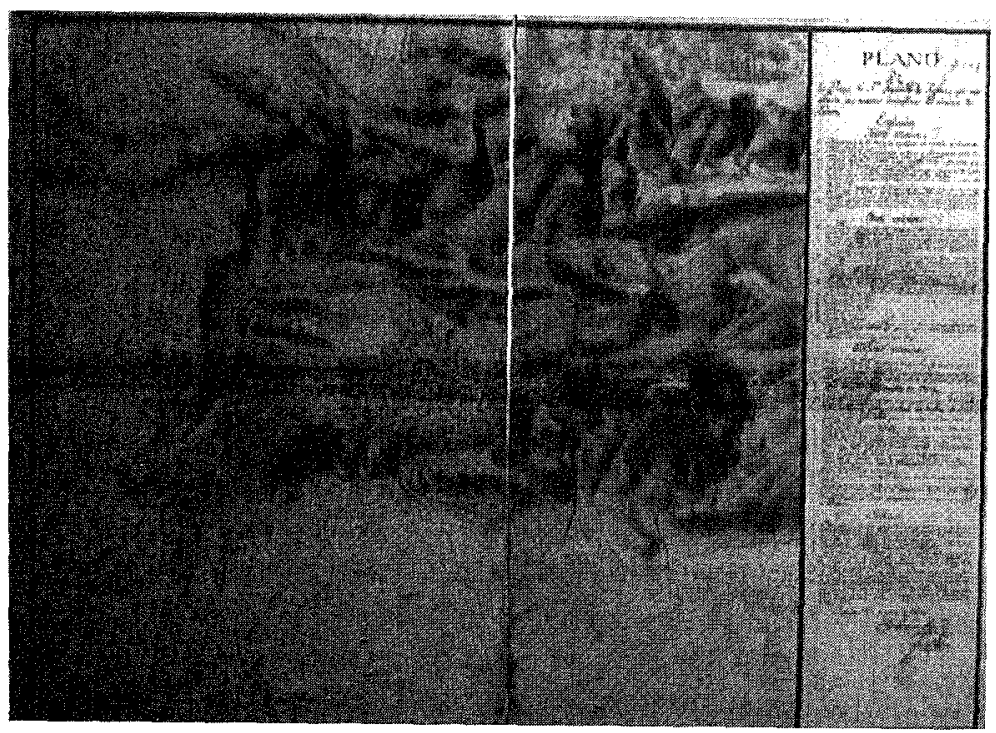

Fig. 4. La fortaleza de San Fernando de Figueras y la villa de Figueras en un plano del ingeniero Antonio López Sopeña datado en 1795 (S.G.E.: Cartoteca Histórica, no 616). 
destacar la cortina oriental del cuerpo de la plaza, en la cual se hallan las caballerizas.

Ante la magnitud de la obra y la previsión realizada con respecto a la guarnición, es lógico que uno de los problemas que se plantearán será el de la imperiosa necesidad de abundantes recursos hídricos. A tal efecto se proyectó una toma de aguas procedente del noroeste que en su último tramo -antes de entrar a la fortaleza a través de la contraguardia de San Pedro- salva la vaguada colindante mediante un acueducto. Es evidente que no sólo la adquisición y traslado de agua suponía la globalidad del problema, sino que otra vertiente del mismo la constituía su almacenamiento. A tal efecto, son numerosas las cisternas (fig. 3) tanto en el cuerpo de la plaza como en las obras exteriores -incluso en el camino cubierto y en el glacis- destacando por su considerable tamaño la situada por debajo del nivel del patio de armas de la fortaleza.

Respecto del proyecto, son varias las conclusiones que podemos obtener. En primer lugar, cabe significar que debido a lo escarpado del frente oriental es precisamente este sector el de menor protección, siendo sus defensas exteriores únicamente dos revellines. Pese a ello, dicho sector se haya reforzado en el cuerpo de la plaza por el baluarte plano de Santa Tecla, intercalado entre las dos obras exteriores.

Es evidente que el sector que ofrecía una mayor preocupación era el occidental, el mejor fortificado de toda la plaza. A partir del cuerpo de la plaza, nos encontramos con el caballero del baluarte de Santa Bárbara. Esta estructura se halla protegida por el hornabeque de San Zenón y los revellines - uno a cada lado- de San Antonio y San José. A fin de reforzar el flanqueo, en la parte posterior de las alas de dicho hornabeque se proyectaron dos reductos. Aparte del revellín que cerraba el hornabeque, un elemento que refuerza aún más la disposición defensiva son las cinco contraminas existentes por debajo del glacis.

Pensamos que la discusión que tenemos que abordar a partir de este momento es clarificar algunas afirmaciones de carácter tipólogico que se han hecho hasta este momento.

No podemos estar de acuerdo con la tipología Vauban con la que Díaz Capmany califica la traza de la fortaleza ${ }^{14}$. Si bien es cierto que algunos rasgos característicos de la plaza parecen tomados de las ideas de este

${ }^{14} \quad$ lbidem, pág. 16. 
ingeniero francés ${ }^{15}$, siendo uno de los más destacables el aprovechamiento del espacio interior de las cortinas, no podemos asignar al corpus teórico de este ingeniero ni la totalidad ni una buena parte de las aplicaciones teóricas llevadas a cabo en el proyecto. En primer lugar, cabe afirmar categóricamente que en la proyección de la obra no se aplica ninguno de los tres sistemas preconizados por Vauban. En segundo lugar, cabe calificar dicha afirmación como reduccionista si contemplamos el hecho de que el tratado de Vauban es de finales del siglo XVII y que durante todo el siglo XVIII se realizan importantísimas aportaciones en este campo ${ }^{16}$.

En el momento de emitir un juicio crítico, son muchísimas las consideraciones que se podrían efectuar, desbordando con creces las pretensiones del presente estudio. Podríamos insistir en consideraciones tales como que nunca se llegara a completar en su totalidad el proyecto, o que la obra manifestara considerables taras, tanto en su emplazamiento como ejecución. Si bien desde el punto de vista del debate teórico, la discusión podría llegar hasta el día del juicio final, en este punto debemos contemplar el carácter que promueve cualquier actuación fortificatoria, que no es otra que la defensa del territorio. Una cosa es el estudio de gabinete, en el cual cualquier maniobra es ejecutada de forma pluscuamperfecta y otra muy distinta es el llevar a cabo tan bellos alardes en el combate real.

En anteriores trabajos hemos expresado - a título de juicio de valorcomo más idóneo el proyecto de Lucuze, sobre todo por las consideraciones globales defensivas con respecto al territorio ${ }^{17}$. Sin embargo, es evidente que cualquier fuerza hostil que quisiera internarse en el territorio catalán se encontraba con el problema de tener que sitiar la plaza trazada por Cermeño, si no quería afrontar serios riesgos en su retaguardia, y es evidente que por muy superado que pudiera estar teóricamente la disposición del sistema defensivo de la fortaleza, dicha operación llevada a la práctica comportaba la desviación de un considerable número de efectivos y una obra de asedio que se podía alargar de forma tan considerable que en muchos casos podria hacer desistir de su intento al atacante.

\footnotetext{
15 Sobre Vauban y sus innovaciones en el campo de la ingeniería militar, de entre la numerosa bibliografía al respecto recomendamos $\mathrm{R}$. BoRnECQUE: La France de Vauban. Arthaud, París, 1984, págs. 19-28.

16 Sobre esta cuestión una fuente importante vuelve a ser A. Marzal. MARTínez, op. cit., t. II págs. 826-1.016. Un aspecto tan importante sobre este particular como la política de traducciones es abordado por J. E. GaRcía MELERo: "Los tratados de arquitectura militar publicados en España durante el reinado de Carlos III", en Espacio, Tiempo y Forma, Ha del Arte 3, Serie VII. Universidad Nacional de Educación a Distancia, Madrid, 1990, págs. 181-223.

17 P. DE l.A Fuente, op. cit.
} 
Prueba de lo dicho es el caso de Rosas, fortaleza del siglo xvı que básicamente será complementada por una serie de obras exteriores a lo largo del XVII - por lo tanto cabe considerarla como una fortaleza anticuada en ese momento- y dominada por una serie de padrastros, que durante la guerra de la Convención y la guerra de la Independencia será un problema considerable para las fuerzas francesas. Paradójicamente, la respuesta de San Fernando no será la misma ${ }^{18}$.

18 Sobre este punto véase C. Diaz Capmanr: op. cit, págs. 69-102. Posiblemente ésta es la mejor aportación que hace este autor en todo su trabajo. 
